\title{
CLEFT LIP AND PALATE CENTRE: THE UNIVERSITY HOSPITAL EXPERIENCE
}

Ghani SHA, Hussain R, Hassan S. et. al. Cleft lip and palate centre: the University Hospital experience. Annals Dent Univ Malaya 1996; 3: 27-37.

\begin{abstract}
The Combined Cleft Clinic at the University Hospital, Kuala Lumpur was organised in 1992. The team consists of Plastic Surgeon, Orthodontists, Speech therapist, Ear, Nose and Throat Surgeon, Audiologist, medical officers and the nurses. We attend the clinic on a regular basis. Specialists from other medical and dental disciplines as well as the members of the Cleft Lip and Palate Association of Malaysia (CLAPAM) do occasionally participate in this set-up. The team members formulate treatment plans for each cleft patient, monitor the patient's growth and development and manage the patient at different stages according to the individual needs. To date, the idea of team approach and an establishment of a centre towards management of cleft patients seem to be the most ideal as the patients are benefiting total treatment and care from various specialists from only one place. The experience of University Hospital as a centre for cleft patients is discussed.
\end{abstract}

Key Words: Cleft Lip and Palate Centre, Team Approach.
Author:

1. Sarah H. A. Ghani B.D.S.(Mal), M.Sc ( Med. Sci. in Ortho.), M.D.O.R.C.P.S (Glasgow)

Co-authors:

2. Rohanah Hussain B.D.S.(Mal), D.D.O.R.C.P.S. (Glasgow).

3. Shafeeq Hasan B.D.S. (Mal).

4. Kim K. Tan M.B.B.S.(Lond), FRCS (Plastic, Reconstructive \& Cosmetic Surgery), (Edin).

5. Mohd. Husni Ahmad M.B.B.S. (Mal)

6. Alasandrie Theseira B.Sc. (Speech Educ.), M.Sc. (Speech Language Pathology), (U.S.A.)

7. Vanaja Venugopal B.Sc. ( Speech Pathology \& Audiology) , (India)

1. \& 2. Lecturers in Orthodontics, Department of Children's Dentistry and Orthodontics, Faculty of Dentistry, University of Malaya, 59100, Kuala Lumpur.

3. Tutor, Department of Children's Dentistry and Orthodontics, Faculty of Dentistry, University of Malaya, 59100, Kuala Lumpur.

4. Plastic Surgeon, Combined Cleft Clinic, University Hospital, Kuala Lumpur.

5. Postgraduate Student, Department of Ear, Nose and Throat, Medical Faculty, University of Malaya, 59100, Kuala Lumpur.

6. Speech Therapist, Rehabilitation Unit, University Hospital, Kuala Lumpur.

7. Audiologist, Rehabilitation Unit, University Hospital. Kuala Lumpur.

\section{Introduction}

Cleft lip, cleft palate or both are among the most common congenital malformations (1). The incidence reported in many parts of the world varies from 0.79 to 3.62 per 1000 live birth (2). In Malaysia, a study was conducted at the Maternity Hospital, Kuala Lumpur in 1966, Stevenson (3) et al reported an incidence of 1.54 per 1000 live birth among Malaysian. In a more recent study by Boo and Arshad in 1990 (4), they reported the incidence to be 1.24 per 1000 live birth. The Chinese had the highest incidence of 1.9 per 1000 birth, while the Malays had the lowest incidence of 0.98 per 1000 birth and the most common type was reported to be the unilateral cleft of the hard and soft palates.

A child with cleft lip and/or palate typically represents a multitude of problems. This fact makes it virtually impossible for any one person to deal with all its aspects and make all the best decisions for the child. For this reason, the team approach is necessary. The interaction and consultation between various disciplines provides care for the cleft patients that can be systematically and comprehensively planned as well as enhances understanding of the possibilities and limitations of various treatment approaches.

The team approach treatment of children with cleft is not new. It was developed in the 1930's in response to the 1933 Social Security Act in the Western countries $(5,6)$. Here in Malaysia, treatment of cleft patients is done in main hospitals with only a few disciplines involved with no standardised protocol and recall appointments for further follow-up. Hence, a number of patients are left untreated or partially treated until adulthood.

In University Hospital, Kuala Lumpur itself, previous attempts have been made at running a combined cleft clinic but died a natural death after a duration of time. In August 1992, initiated by Associate Professor Dr. Kim K Tan, the plastic surgeon, the Combined Cleft Clinic at the University Hospital was revived.

This paper is aimed at reporting the experience of the University Hospital in functioning as a Cleft Lip and Palate Centre as well as providing guidelines and treatment protocol for the management of facial cleft deformaties.

\section{COMBINED CLEFT CLINICS}

Our main treatment objectives are to improve facial 
aesthetics, oral functions and speech, and to achieve a stable occlusion. These are all important aspects of enhancing the patient's well-being and self-esteem. The team members are made up of Plastic Surgeon, Orthodontists, ENT Surgeon, Speech therapist, Audiologist, medical officers, and staff nurses. They meet regularly for consultation, assessment and treatment planning for the cleft patients. The members meet on every first Thursday of the month. Occasionally, a representative of parents support group (CLAPAM), tutors and specialists from other medical and dental disciplines do also participate in this clinic.Consultation and treatment by the other specialists may be called upon when required. The team handles approximately fifteen patients per session in the clinic. The centre gets referrals from the regional hospitals all over Malaysia.

Cases are presented (for new and referred cases), reviewed and discussed to decide on the next course of treatment required. Patients records are updated according to the clinic protocol or checklist of the individual specialty of the team. As an example, a patient may need new radiographs, study models, speech assessment, hearing test, dental reviews and surgical appointments. The timing of various disciplines contributing towards total treatment of the cleft patients is indicated in Table 1, however, individual needs vary and adjustments may be required in the timing and the delivery of certain procedures.

\section{FUNCTIONS}

The team fulfills 4 main functions, namely:

1. By co-operating together, the various members of the team facilitate each other's efforts in the treatment of the condition. As such, the clinical work is made easier by one member of the team preparing the case for an operation by a second member.

2. It provides a mechanism for recording by various means, all the treatment carried out on the patient. This includes recording the child's growth and development as well as the results of the various procedures performed on the patient.

3. The structure of the team provides an ideal training mechanism whereby undergraduate and postgraduate students can be taught the basics of cleft lip and palate management. At a higher level, the junior members of the team can work with the senior members whilst developing their treatment skills.

4. In the combined clinical meetings and combined consultation, the set-up provides an opportunity for the new parents with cleft babies to meet not only the the professional team members, but also other CLAPAM members, for advice, counselling and exchange of views and ideas.

\section{THE TEAM MEMBERS}

The Plastic Surgeon

The plastic surgeon is the chief coordinator of the combined cleft clinic. His role starts early in the initial stage after the birth of a cleft child where he visits and councels the parents. Clearly this is a traumatic time for the parents and an initial visit by the surgeon will allay some of their anxiety, guilt and more importantly provides the parents information on the treatment plan of the patient $(7,8)$.

Feeding can be a problem for infants with cleft of the hard palate. In the majority of cleft babies, bottles with special teat or spoon are found to be suitable for feeding (Figure 1a \& 1b).It is not necessary to institute tube feeding to cleft babies. Frequently, a visit from a member of the support group (CLAPAM) is arranged and this can be of great help to the parents as they will have first hand information about feeding and what the treatment entails.

The plastic surgeon operates three to four cases a

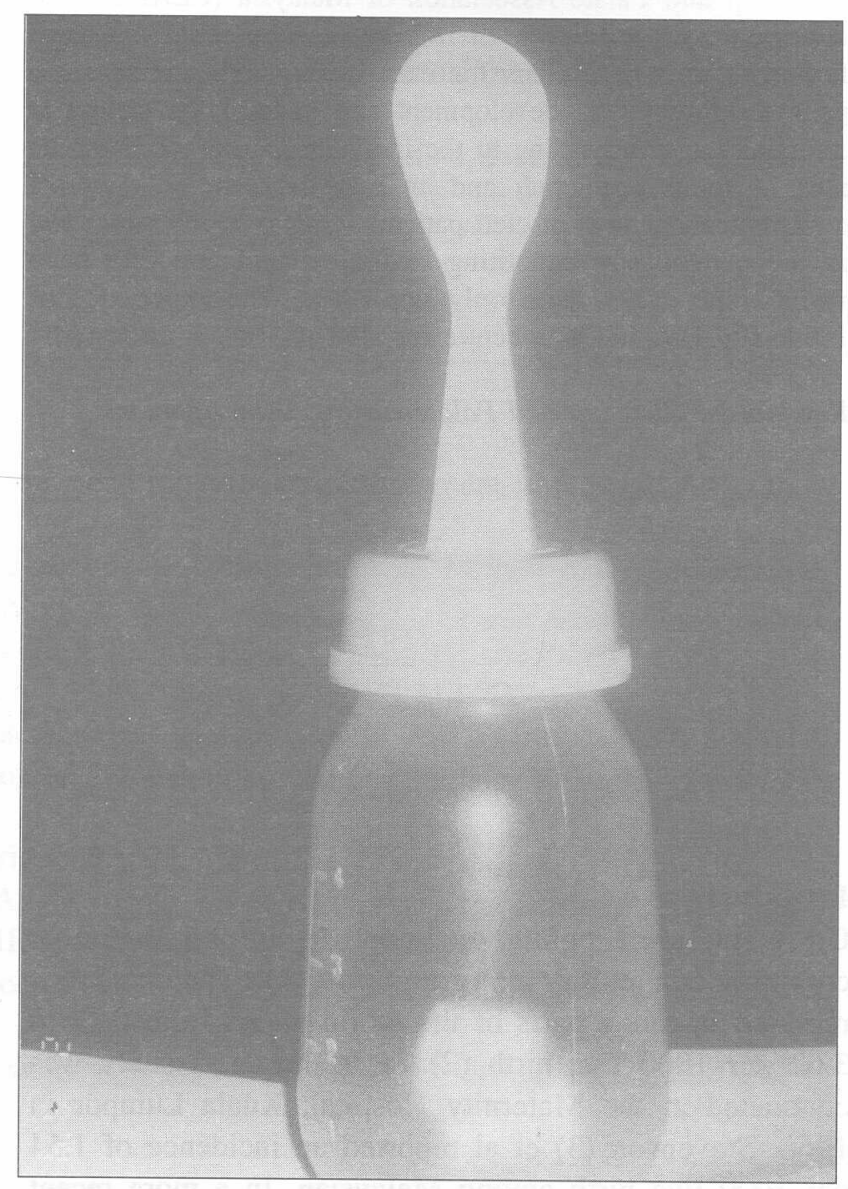

Figure 1a: A special spoon attached to the feeding bottle.

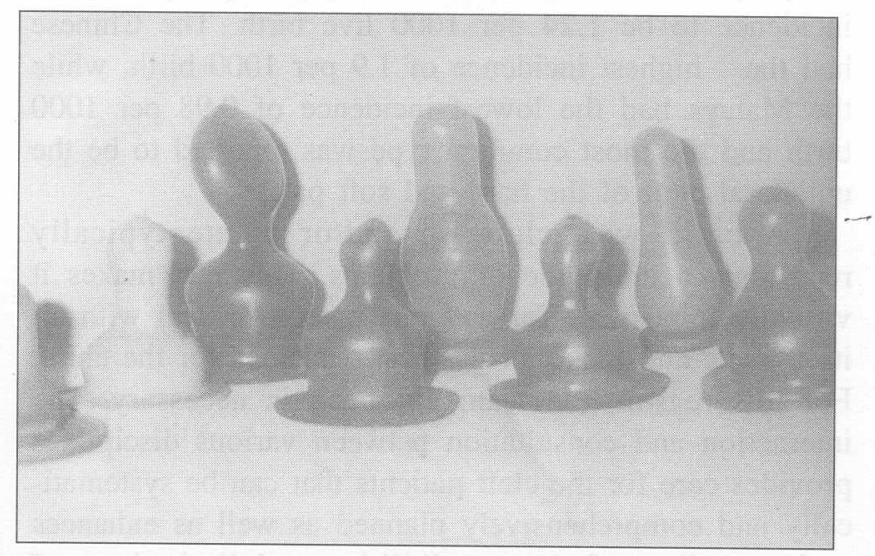

Figure 1b: Different types of special teats for cleft babies. 


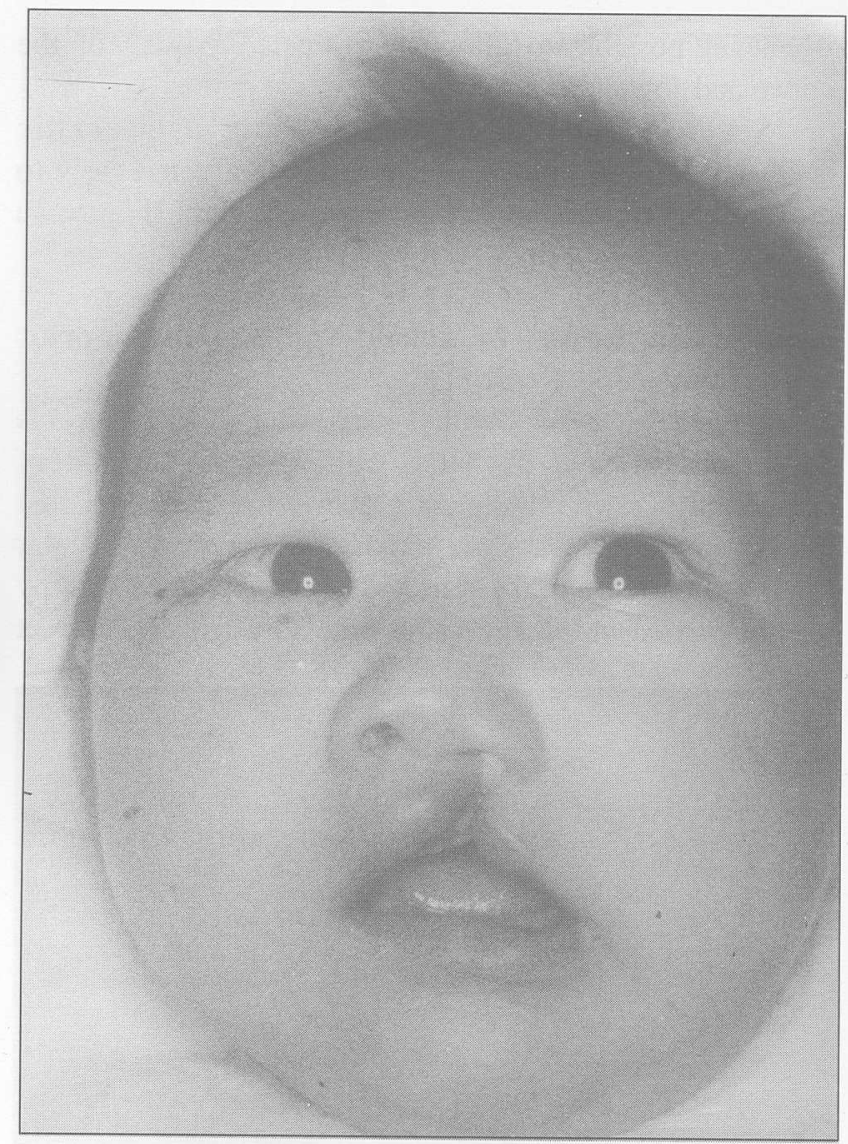

Figure 2a: Before lip surgery at 3 months of age

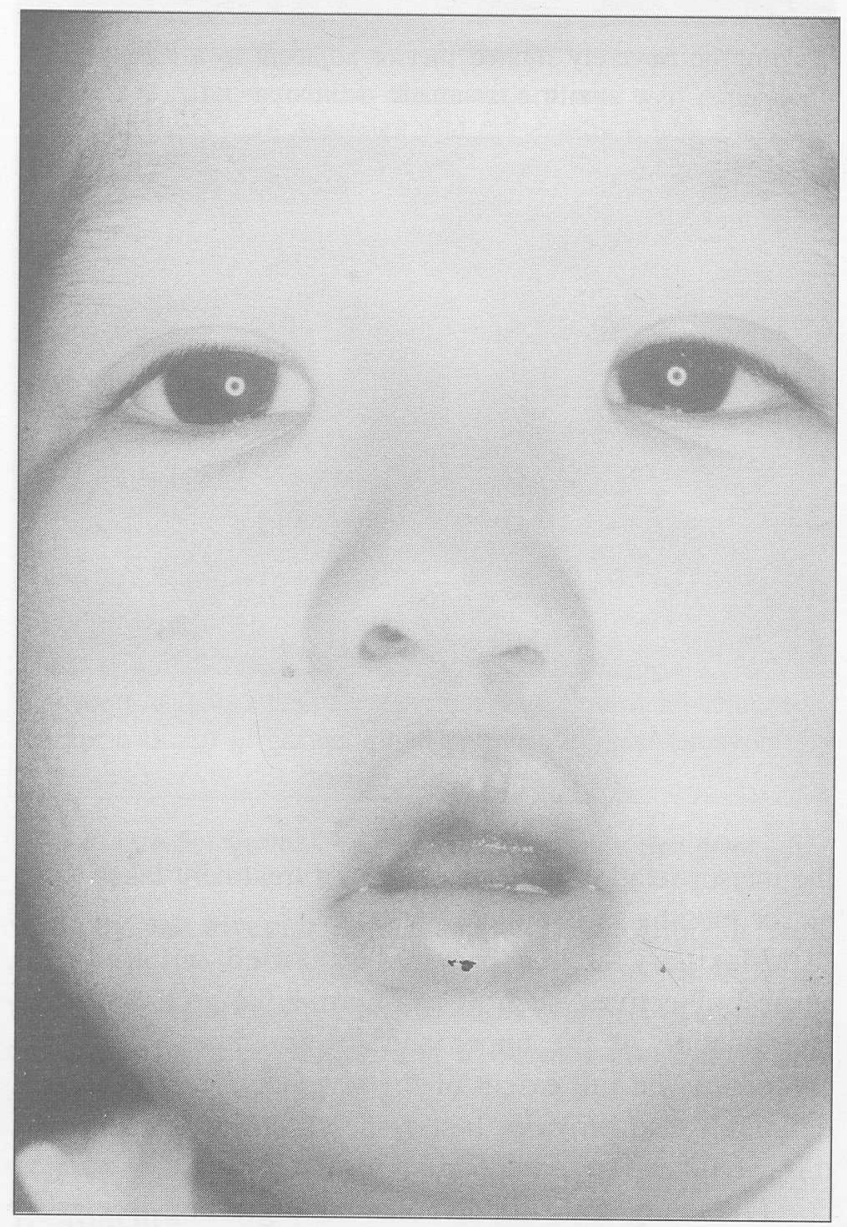

Figure 2b: After lip surgery at 9 months of age
week.Normally, the surgical repair is carried out in stages. Surgical closure of the lip is usually scheduled around three months of age. At this time the infant is healthy enough to ensure a safe procedure and uncomplicated healing. In certain suitable cases, if requested by the parents, the lip can be repaired at birth.The nose is also corrected at the time of the lip repair. The objective of this surgery is to close the cleft early and produce a lip of adequate length that is functionally satisfactory. The child will achieve a pleasing apprearance as well as allowing the face to develop normally (Figure 2a \& 2b). The palate is usually repaired at six months of age. There are wide variations in both the timing and the technique of surgical repair $(8,10)$.

Palatal closure may involve more than one surgical procedure. The goal of the palatal surgery is to close the cleft so that the palate can function normally during eating and drinking as well as providing sufficient length and movement of the palate to ensure normal speech production.The cleft in the alveolar region is usually left until bone grafting stage.Around the age of five, a 'Touch-up' surgery to the lip or nose can be performed to give the patients the best aesthetic results before they start school. This is also the time to assess some of the patients with velopharyngeal incompetence (VPI). This procedure is done with the help of naso-endoscopy to decide on the need of any surgery of the soft palate. Various types of pharyngoplasty can be performed according to the findings of the naso-endoscopy. The next essential age for the surgical intervention is at the age of 10-11 years. Following orthodontic arch expansion, a bone graft is placed in the alveolus to improve the arch form and establish an intact dental arch. This would also allow eruption of the canine which is frequently displaced in the cleft region. Alveolar fistula is also corrected at this stage. This bone grafting procedure can also be managed by an oral surgeon.

After puberty, there is usually a request from patients for more "touch-up" surgery. This may take in the form of rhinoplasty, lower lip reduction and upper lip revision and is often coordinated with orthognathic surgery if required. The oral surgeon is consulted for treatment planning and management.

\section{The Orthodontist and other Dental Specialists}

The preservation and maintenance of the dentition at this early stage is paramount. Almost all cleft lip and palate children will have dental anomalies which may include missing permanent teeth, supernumerary and/or supplemental teeth, teeth of abnormal marphology, hypoplastic teeth, delayed or abnormal eruption and malocclusion. As early as possible after birth, here in the cleft set-up, the paedodontist takes the responsibility of instituting a preventive programme such as proper tooth brushing techniques, diet counselling on the importance of low intake of sugar-containing foods and drinks as well as early provision of restorative treatment if dental caries develops. For patients who come from other places, they are advised to seek treatment with the local dental 
officers or private dental practitioners.

Orthodontic intervention of cleft management comes in several phases that closely parallel the various stages of growth and development. Short bursts of active treatment are followed by periods of inactivity. Each phase of treatment therapy is kept short so as not to exhaust the patient's patience and enthusiasm. In general the orthodontic intervention can be divided into the following phases.

\section{Birth to three months - Pre-surgical Oral Orthopaed- ics}

A feeding plate (12) or orthopaedic appliance (13) may be given when indicated. Extra-oral strapping can be used to provide an orthopaedic force to align displaced segments before lip repair for some severe cleft patients $(12,15)$. Figure $3 a \& 3 b$.

\section{Seven years old - Interceptive Treatment during Early Mixed Dentition \\ Orthodontic treatment with simple appliances may be carried out if the developing malocclusion causes a functional disturbance (14). The two most common problem needing attention at this stage are the develop- ing anterior cross-bite and rotated upper incisor adjacent to the cleft area. Anterior cross-bite associated with a mandibular displacement should be corrected early}

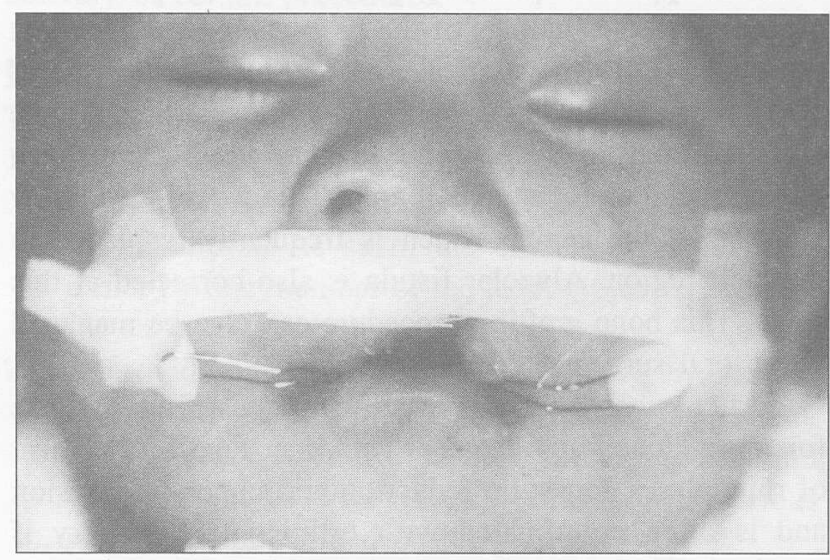

Figure 3a: Extra-oral strapping may be necessary to provide an orthopaedic force and align severe displaced segments.



Figure 3b: Orthopaedic plate with $0.5 \mathrm{~mm}$ stainless steel wire for retention whenever possible to allow co-ordinated growth of the upper and lower jaws.

Severely rotated incisors causing a traumatic occlusion to the lower teeth need to be corrected early to prevent periodontal damage to the lower teeth (Figure 4a $\& 4 b)$.

\section{Eight to Nine years - Maxillary Arch Expansion prior to Alveolar Bone Grafting.}

A Quadhelix is used for arch expansion (9 \&.12). This appliance is made of bands soldered to $0.9 \mathrm{~mm}$ of stainless steel wire with coils and cemented onto first molars to produce a slow expansion by dento-alveolar tipping (Figure 5a \&5b). Limited fixed appliance therapy maybe carried out at the same time to align and correct

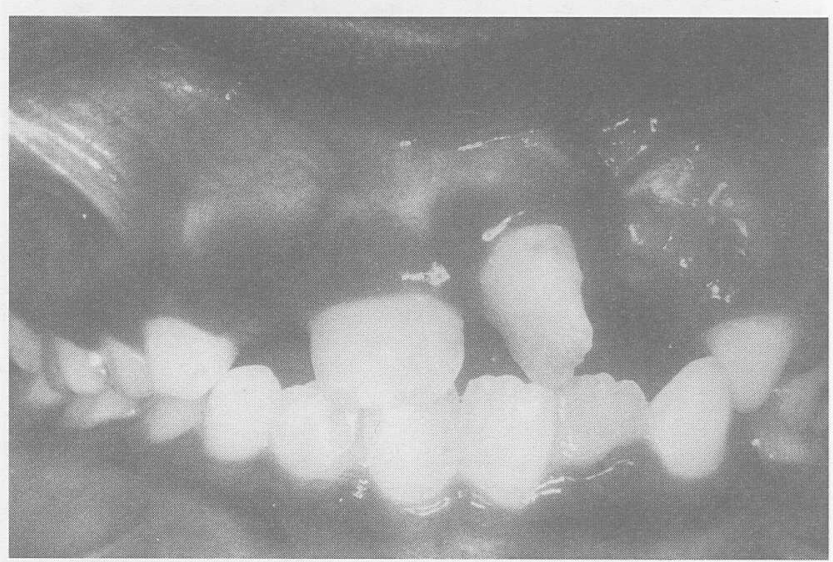

Figure 4a: Severely rotated incisor adjacent to a cleft region with a traumatic occlusion

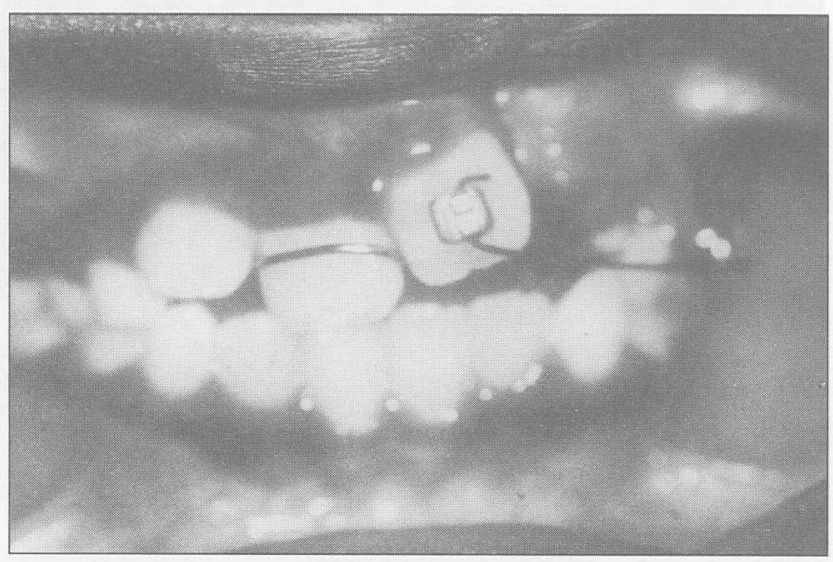

Figure 4b: Simple interceptive treatment using an upper Removable Appliance with a 'whip' spring to derotate upper left central insisor

the incisor relationship. This phase of treatment takes three to six months to complete.

Maxillary arch expansion is carried out to fulfill several objectives, such as to improve the arch form and coordination of the upper and lower arches. With this procedure, the full extent of the alveolar defect is clearly defined.It also provides better access during surgery and this would ensure complete surgical closure.

Following that, the alveolar bone grafting is carried out. Alveolar bone grafting (16) is a surgical procedure 


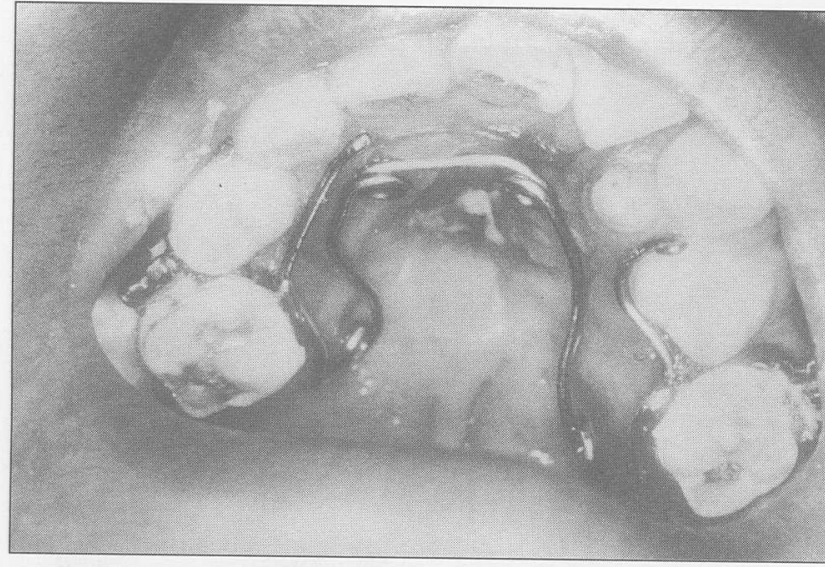

Figure 5a: Maxillary Arch Expansion prior to alveolar bone grafting using a Quadhelix.



Figure 5b: After 6 months, the maxillary was well expanded; premolar teeth were derotated and the dentition was generally aligned.

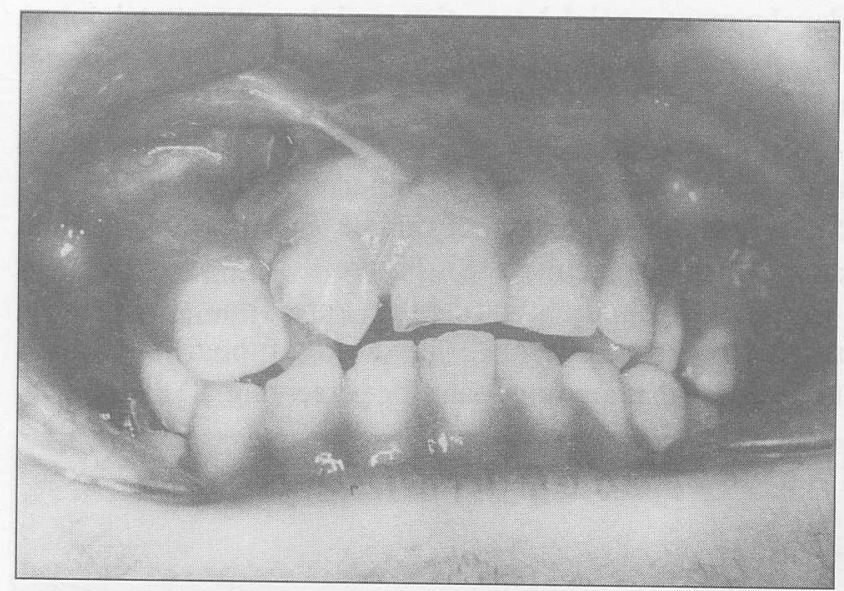

Figure 5c: Frontal View - Pre-orthodontic treatment

whereby the alveolar defect is closed by filling it with cancellous bone chips to restore the tooth bearing function. It is normally done to allow eruption of the upper canine in the cleft region and to form a continuous and stable arch (Figure 6a \& 6b).

\section{Twelve to fourteen years - Definitive Orthodontic} Treatment

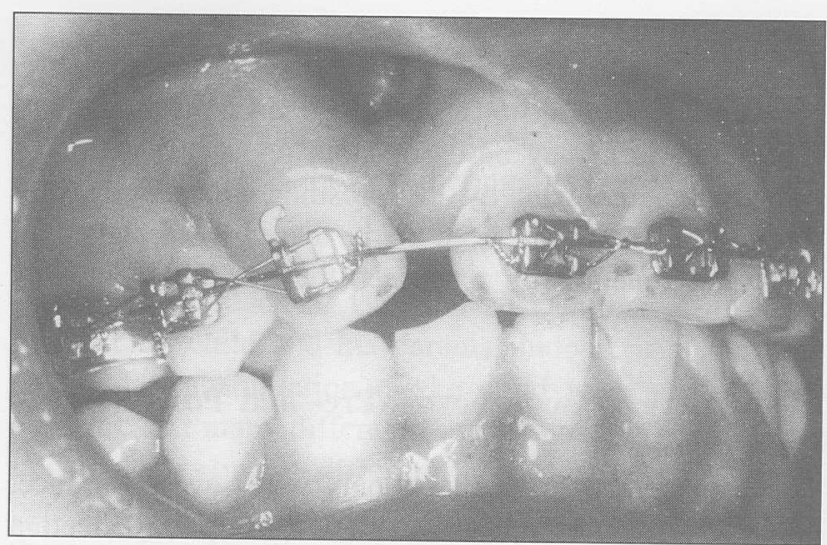

Figure 5d: Frontal View - Orthodontic alignment completed; space created for better assess during surgery and ensure complete surgical closure and bone grafting procedure

Definitive orthodontic treatment maybe carried out at this stage for cases not indicated for orthognathic surgery. The objectives of treatment are mainly to relieve crowding, alignment of teeth, space closure, centre line correction as well as coordination of upper and lower arches to achieve a normal occlusion.


Figure 6: Upper left canine of a cleft patient.

a) Prior to bone grafting. Canine is frequently displaced and unable to erupt into the arch

b) Post bone grafting. Eruption of the upper left canine observed. 
Fixed appliance is used.The duration of treatment is between 18 months to 2 years. Advance restorative or prosthetic work may be carried out upon completion of orthodontic treatment by the prosthodontist. Bridges or removable prosthesis need to be carefully designed to serve the function of a retainer for the orthodontically corrected teeth.

Eighteen years and above - Pre- and Post- Surgical Orthodontics.

The management of cleft patients with severe midface, anterior-posterior and/or vertical discrepancy need a combined surgical-orthodontic approach. Orthodontic treatment is divided into two phases, pre- and postsurgical orthodontics.

Pre-surgical orthodontics would involve alignment and levelling of teeth, arch co-ordination and decompensation of the upper and lower dentition. Post-surgical orthodontics is carried out after orthognathic surgery. This short phase of orthodontic treatment is necessary for final tooth positioning to achieve good interdigitation and to close any residual space.

\section{The Speech-Language Therapist}

The speech-language therapist first becomes involved with children with cleft palates during the child's first year. These early sessions are to monitor the child's language development and articulation of speech sounds periodically. Early intervention is needed so that good speech habits are acquired before the child learns to speak.

When the child has adequate expansive language, an articulation assessment is carried out to determine if the child is able to make the speech sounds correctly. Children may make errors such as omitting, substituting or distorting specific sounds. Speech therapy is provided to correct misarticulations as well as prevent undesired compensatory substitutions such as nasal emission and glottal stops. The speech-language therapist needs to work closely with the family so that they are able to practise the activities at home (6).

The child also has to direct airflow orally in order to attain appropriate resonance and eliminate nasal escape (Figure $7 \mathrm{a} \& 7 \mathrm{~b}$ ). Besides perceptual measures of oral-nasal balance, the velopharyngeal closure competence can be evaluated more objectively using naso-endoscopy,

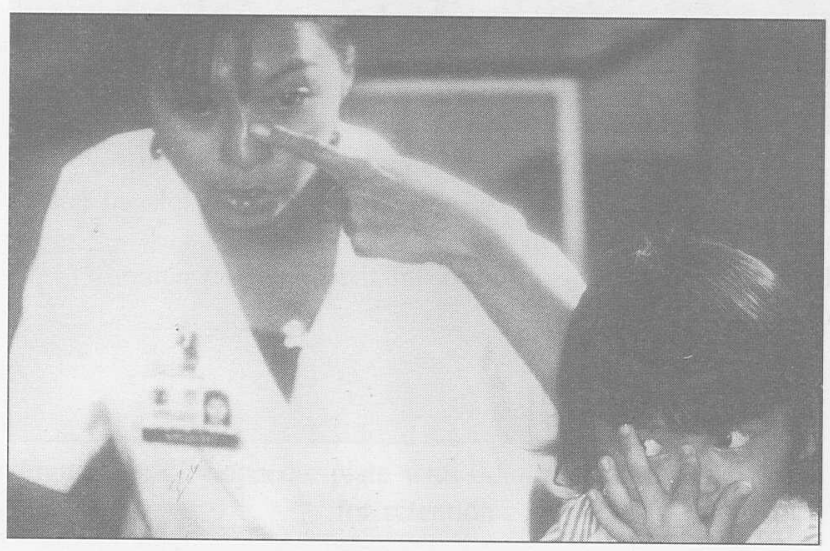

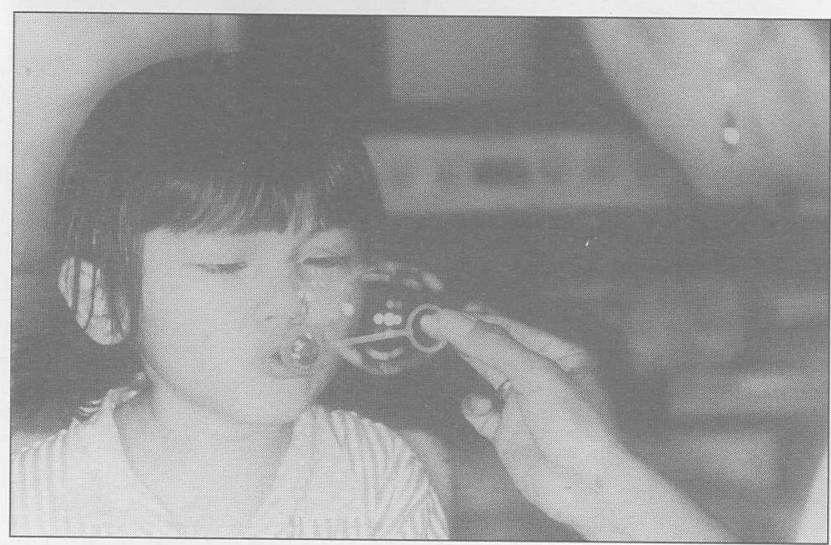

Figure 7a \& 7b: The. speech-language therapist penform activities such as "bubble-blọwing" to direct airflow orally in order to attain appropriate resonance.

videofluoroscopy and air-flow measurements. Individuals who show clear evidence of achieving velopharyngeal closure may undergo speech therapy for hypernasality.

However, those with velopharyngeal inadequacy will be referred to the combined clinic for further management (17).

\section{The Audiologist and ENT Surgeon}

An audiologist is a professional trained in the diagnosis and rehabilitation of all non-medical aspects of hearing and hearing disorders. The audiologist assesses hearing as a routine part of the ongoing management of the child with cleft palate as they are more prone to middle ear problems than the normal population.Hearing impairment, depending on the type and degree, age of onset and other factors have direct impact on speech and language development, social interaction and progress at school.

Audiological assessment for infants and young children below 5 years are based on Behavioral Audiometry tests, Play Audiometry and objective assessments using Acoustic Impedence tests and Brain Stem Audiometry to assess middle ear function as well as sensorinueral acuity (18). Figure 8.

In older children and adults, Pure Tone Audiometry tests can determine the threshold of hearing at the frequencies that are important for perceiving all the speech

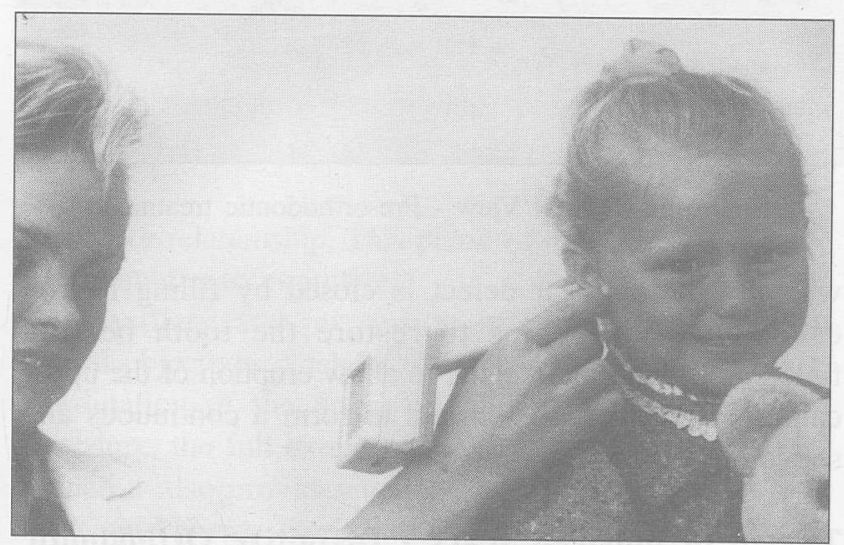

Figure 8: Behavioral Audiometry Test and Play Audiometry Test are used for children below 5 years to assess middle ear function 
sounds adequately. Prior to the cleft lip or palate surgery, an audiological assessment with tympanometry is done to determine the status of the middle ear system. The child can develop a middle ear condition, otitis media with effusion (OME). This is a condition where there is an exudation of fluid from blood and lymphatic vessels into the middle ear due to inequality of pressure within the middle ear and the atmospheric pressure (20). It can result in a conductive hearing loss, whereby the child receives the speech signal at a reduced level. Once the tests are complete, the case is referred to the Ear Nose and Throat specialist for assessment and management, and further follow-up at the combined cleft clinic.

As far as the medical treatment is concerned, surgery remains as the mainstay of treatment for otitis media with effusion (OME) secondary to cleft palate. This is in the form of myringotomy with insertion of ventilation tube, together with palatal repair.

\section{Cleft Lip and Palate Association of Malaysia (CLAPAM)}

Many parents who have a baby with a cleft lip, cleft palate, or both feel isolated and alone.They often say that it is very helpful to be able to talk to other parents of children with clefts.In many parts of the world there are local parents support groups. These support groups are organised by parents of children with clefts and adults with clefts who meet to share common concerns and ideas and because these individuals share similar experiences, they prove to be a continuing source of support for each other. Here in Malaysia, CLAPAM was formed in November 1993. Since then, members who are parents of children with clefts, adults with clefts, professionals and specialists as well as volunteers formed an active team (Figure 9). Information is given out through pamphlets in most hospitals in the country for the newborns with clefts. The members volunteer to provide counselling for parents and assist newborn cleft babies with feeding problems (7).

The committee members organise meetings once in every two months where professionals from various fields are invited to deliver talks to the new and existing members of CLAPAM.

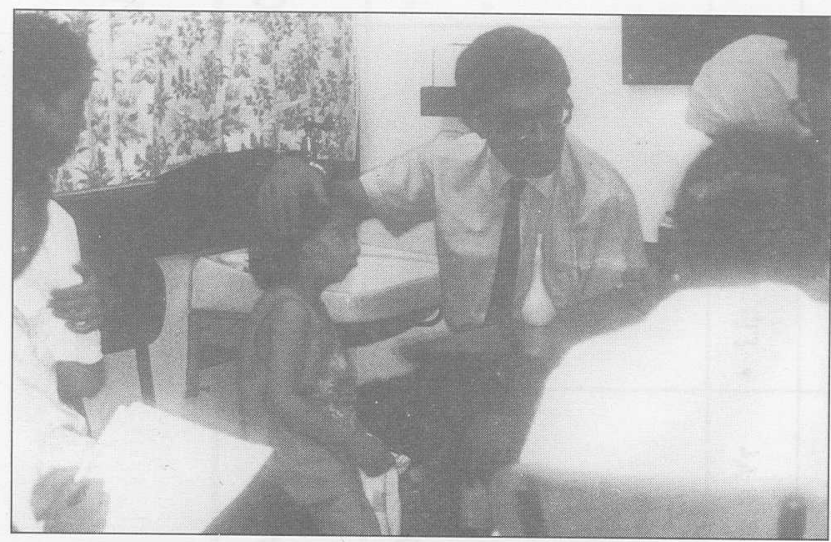

Figure 9: The Combined Cleft Clinic at University Hospital, K.L.
Specialists such as the plastic surgeon, orthodontist, ENT surgeon, audiologist, speech therapist, nutritionist, dietician, counselors and parents share their experience and deliver talks at these gatherings.

\section{CONCLUSION}

The team approach is a logical answer to the problems of the cleft child and his parents. Indeed, it is the only one. Total rehabilitation of the child with a cleft is so complex that many different aspects must be brought into unison by as many different specialists. Not all team personnel, however, spring into action at the same time; there are, in practice, what may be termed functional subgroup(subteams) acting concertedly. Rehabilitation is progressive, not static; each age period has its unique set of problems of adjustments and full participation from all members is required at all times to tackle the situations correctly.

Three years had elapsed since the formation of the centre, like all things in life, this centre of course, is not without its problems. The most difficult task in running a multidisciplinary clinic is to maintain the participation of all disciplines in the activities of the clinic. This is due to firstly, the on-going change in the team members involved. Some of the members have left the group for private practice or were transferred elsewhere which made the follow-up treatment slightly delayed and in addition to that the existing members are currently overloaded with cases. We, however, are fortunate to be able to see patients on a regular basis still with the continuous effort of the co-ordinator who comes on a part-time basis to run the clinic and operate on the certain cleft cases. Secondly, the lack of initiative of some members to determine their particular roles and duties in such a multidisciplinary setup.

Apart from being a treatment providing centre, this centre also function as a base for exchange of education and research programmes with other centres abroad. Occasionally visiting Consultants from Australia, United Kingdom, United States, India, Singapore and other neighbouring countries are invited to deliver lectures on current trends. Discussions focus on diagnosis and treatment planning of difficult cases. They also perform operations and demonstrate the latest surgical methods and techniques.

To date the team has been successful in carrying out its duties to patients mainly with the cooperation of the patients themselves, parents, team members and staff of the hospital. A single, integrated, comprehensive treatment plan is set up by this team, and each cleft patient is carefully documented under a rigorous protocol at every phase of treatment and development to obtain longitudinal data. The accumulated data of these patients provide optimal surgical timing and the effects of surgery on growth and development.

This centre would also serve as a model for future planning of other centres and a potential base for research and further specialists training. Lastly, it is our hope and 
aim that through our concerted efforts, our cleft children would grow into normal healthy adults and will integrate well into the society.

\section{Acknowledgement}

The author wishes to thank the team members for their contributions to this paper and the on-going care for our cleft patients.

\section{REFERENCES}

1. Carter CO. Genetics of common single malformations. Br. Med. Bull 1976; 32: 21-6.

2. Vanderas A. Incidence of cleft lip, cleft palate and cleft lip and palate among races. A review. Cleft Palate J 1987; 24: 216-25.

3. Stevenson AC, Johnston HA, Stewart MIP, Golding DR. Congenital malformations. A report of study of series of consecutive births in 24 centres.

Bull WHO 1966; 34 (supp11) :47-54.

4. Boo NY, Arshad AR. A Study of cleft lip and palate in neonates born in a large Malaysian maternity hospital over a 2-year period. Sing. Med J 1990; 31: 59-62.

5. Neiman GS, Haas DG. The Team Approach for treatment of cleft lip and palate. Ohio Dent J 1988; 62(4):17-20.

6. Brogan WF. Team Approach to the treatment of cleft lip and palate. Annals Academy of Med. July 1988; 17 (3): 335-8.

7. Cleft Lip and Palate Assoc. of Malaysia (CLAPAM). Personal communication from The President, CLAPAM, 22. Jln SS 3 / 74, Tmn University 47300 P.J.

8. Mars M. Clefts of the lip and palate. In: Houston WJB, Stephens CD, Tulley WJ ( 2nd.ed). A Textbook of Orthodontics. Wright 1992; 392-3.

9. Thom AR. Modern management of cleft lip and palate patient. Dental Update. Dec. 1990; 402-8.

10. Lydiatt DD, Yonkers AJ, Schall DG. The management of the cleft lip and palate patient. Nebraska Med. J. Nov 1989; 325-9.

11. Kurt WB. Treatment of facial cleft deformaties. An illustrated guide (1st ed). Ishiyaku Euro America Inc. 1995; $1-12$.

12. Shaw WC, Semb G. Current approaches to the orthodontic management of cleft lip and palate. J of the Royal Society of Medicine, Jan 1990; 83: 30-3.

13. Hochban W, Austermann KH. Pre-surgical orthopaedic treatment using hard plates. J. of Cranio-Max-Facial Surg.
$1989 ; 17: 2-4$.

14. Shaw WC. Orthodontic treatment of malocclusion associated with repaired complete clefts of lip and palate. Br. J. Ortho. 1979; 6: 73-83.

15. Shaw WC. Early orthopaedic treatment of unilateral cleft lip and palate. Br.J. Ortho. 1978; 5: 119-32.

16. Freihofer HPM, Kuijpars-Jagtman AM. Early secondary ostoeplastic closure of the residual alveolar cleft in combination with orthodontic treatment. J. Cranio-Max- Facial Surg. 1989; 17: 26-7.

17. Theseira A. Personal Communication. The Rehabilitation Unit, University Hospital, K.Lumpur.

18. Grant HR. Cleft palate and glue ear. Archives of Diseases in Childhood.1988;63: 176-9.

19. Venugopal V. Personal Communication. The Rehabilitation Unit, University Hospital, K.Lumpur.

20. Husni A. Personal Communication. The ENT Dept. University of Malaya, K.Lumpur. 


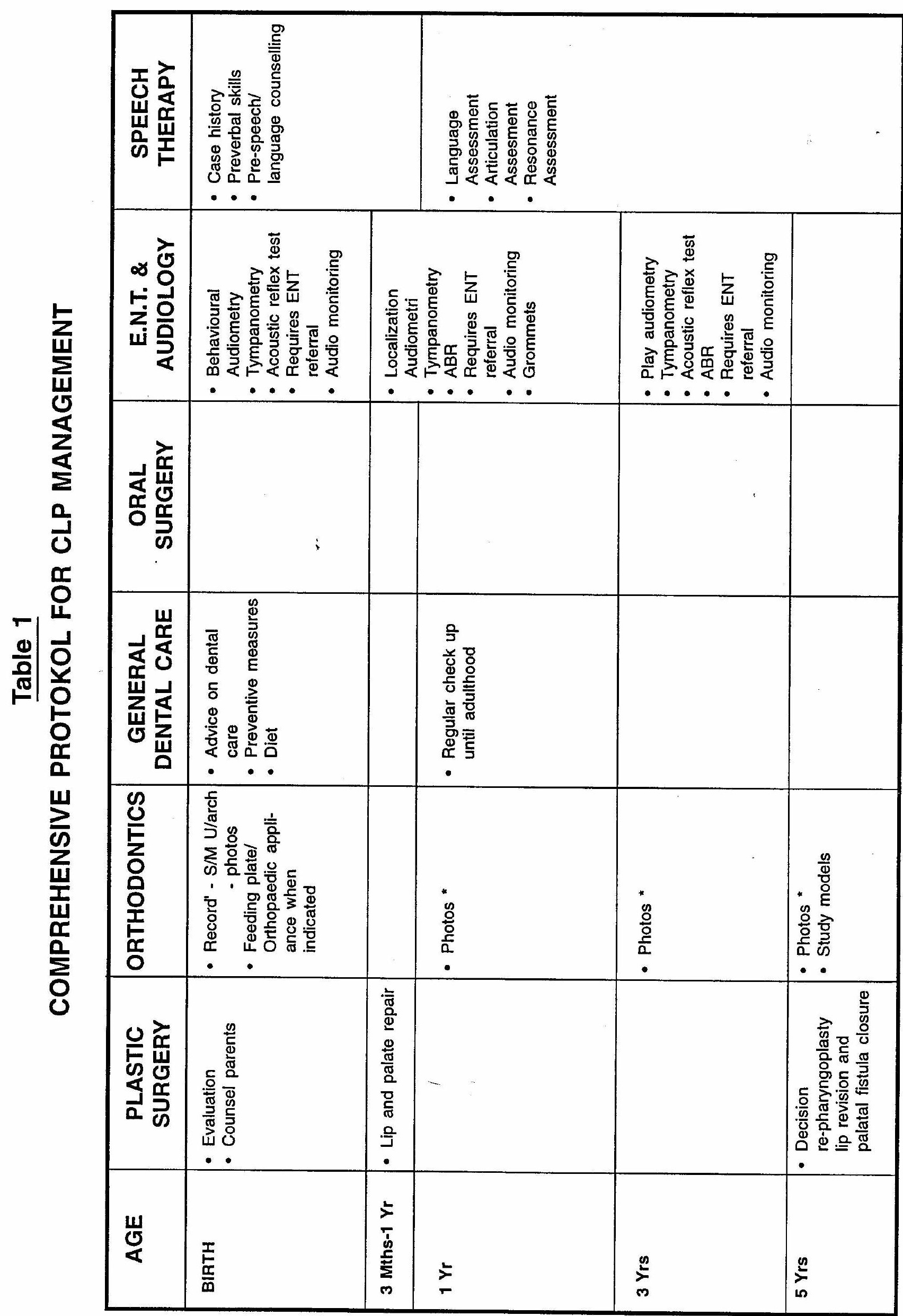




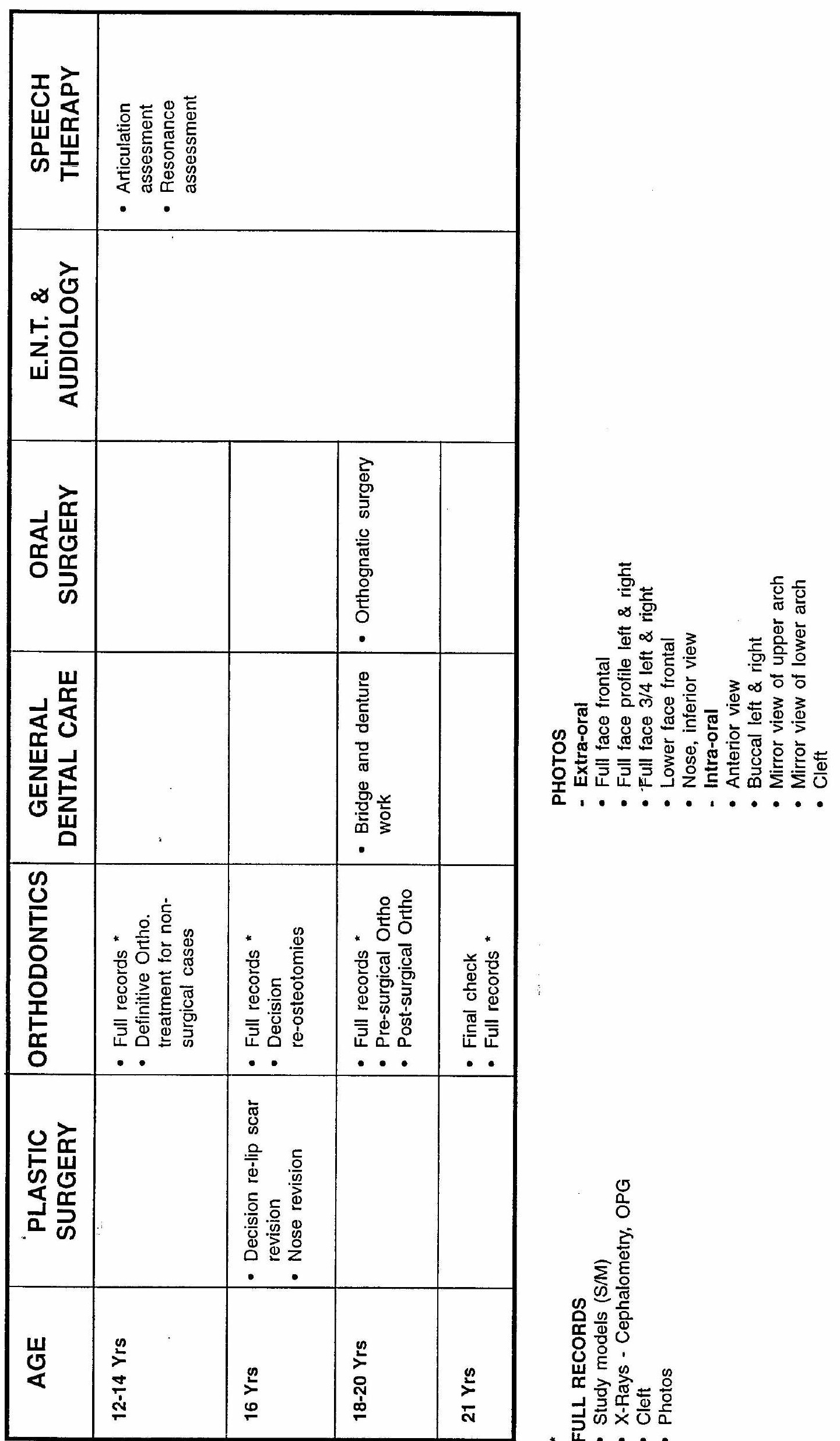




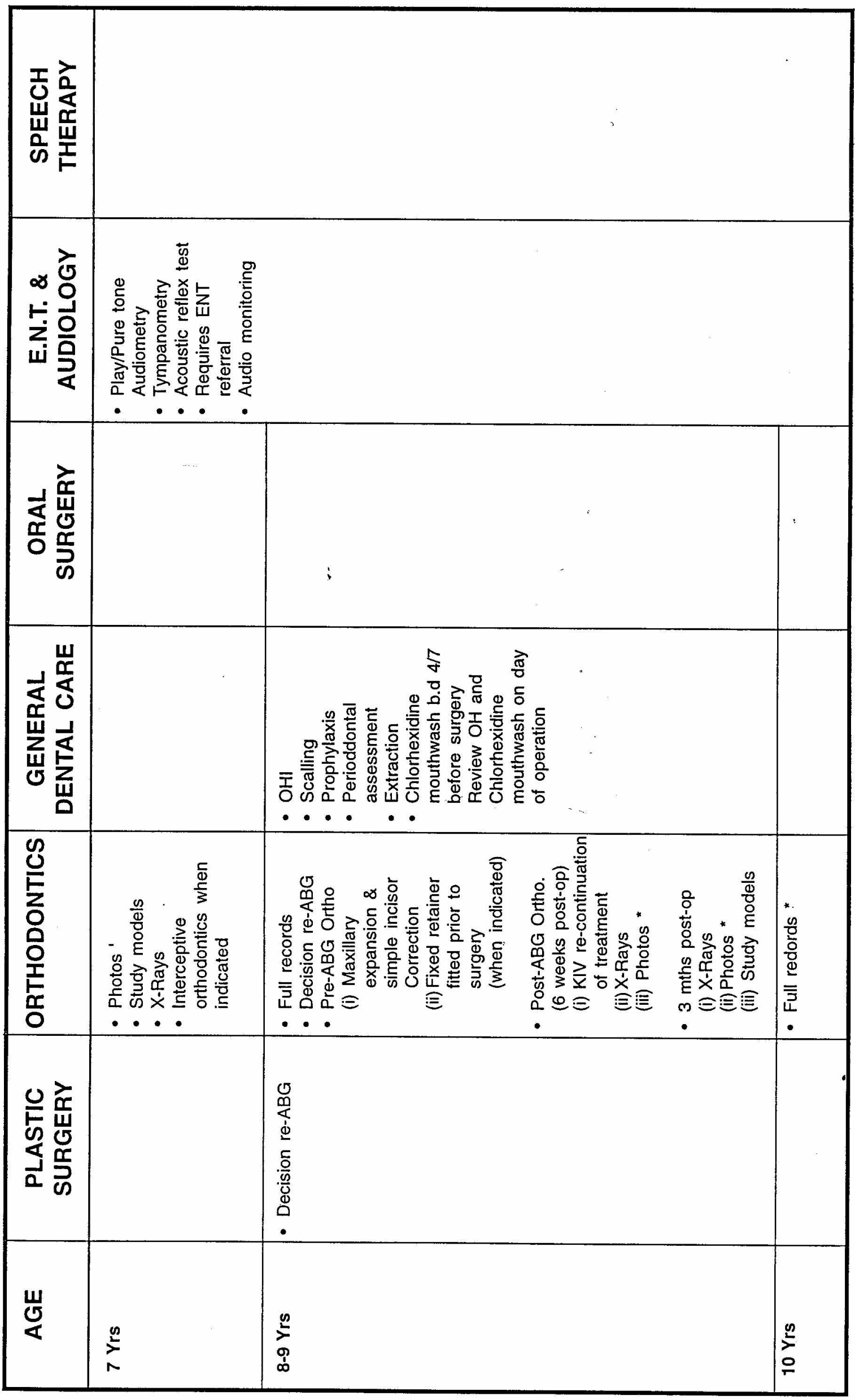

